\title{
S100a4 upregulation in Pik3caH1047R;Trp53R270H;MMTV-Cre-driven mammary tumors promotes metastasis
}

\author{
Wenlin Yuan ${ }^{1}$, Leonard D. Goldstein ${ }^{1,2}$, Steffen Durinck ${ }^{1,2}$, Ying-Jiun Chen ${ }^{1}$, Thong T. Nguyen ${ }^{1}$, Noelyn M. Kljavin³,
} Ethan S. Sokol ${ }^{4}$, Eric W. Stawiski ${ }^{5}$, Benjamin Haley ${ }^{1}$, James Ziai ${ }^{6}$, Zora Modrusan ${ }^{1}$ and Somasekar Seshagiri ${ }^{17^{*}}$ (D)

\begin{abstract}
Background: PIK3CA mutations are frequent in human breast cancer. Pik3caH1047R mutant expression in mouse mammary gland promotes tumorigenesis. TP53 mutations co-occur with PIK3CA mutations in human breast cancers. We previously generated a conditionally activatable Pik3caH1047R;MMTV-Cre mouse model and found a few malignant sarcomatoid (spindle cell) carcinomas that had acquired spontaneous dominant-negative Trp53 mutations.

Methods: A Pik3caH1047R;Trp53R270H;MMTV-Cre double mutant mouse breast cancer model was generated. Tumors were characterized by histology, marker analysis, transcriptional profiling, single-cell RNA-seq, and bioinformatics. Cell lines were developed from mutant tumors and used to identify and confirm genes involved in metastasis.

Results: We found Pik3caH1047R and Trp53R270H cooperate in driving oncogenesis in mammary glands leading to a shorter latency than either alone. Double mutant mice develop multiple histologically distinct mammary tumors, including adenocarcinoma and sarcomatoid (spindle cell) carcinoma. We found some tumors to be invasive and a few metastasized to the lung and/or the lymph node. Single-cell RNA-seq analysis of the tumors identified epithelial, stromal, myeloid, and T cell groups. Expression analysis of the metastatic tumors identified S100a4 as a top candidate gene associated with metastasis. Metastatic tumors contained a much higher percentage of epithelial-mesenchymal transition (EMT)-signature positive and S100a4-expressing cells. CRISPR/CAS9-mediated knockout of S100a4 in a metastatic tumor-derived cell line disrupted its metastatic potential indicating a role for S100a4 in metastasis.

Conclusions: Pik3caH1047R;Trp53R270H;MMTV-Cre mouse provides a preclinical model to mimic a subtype of human breast cancers that carry both PIK3CA and TP53 mutations. It also allows for understanding the cooperation between the two mutant genes in tumorigenesis. Our model also provides a system to study metastasis and develop therapeutic strategies for PIK3CA/TP53 double-positive cancers. S100a4 found involved in metastasis in this model can be a potential diagnostic and therapeutic target.
\end{abstract}

Keywords: Pik3caH1047R, Trp53R270H, S100a4, Mammary tumors, Breast cancer, Metastasis

\section{Background}

PIK3CA gene mutation or amplification occurs in 26$36 \%$ of breast cancers $[1,2]$. The most recurrent PIK3CA mutation, H1047R, is constitutively active and promotes PI3K signaling [3]. Several mouse transgenic or knock-in models have been developed to study PIK3CA-driven

\footnotetext{
* Correspondence: sekar@sgrf.org

'Department of Molecular Biology, Genentech Inc, 1 DNA Way, South San Francisco, CA 94080, USA

${ }^{7}$ SciGenom Research Foundation, Bangalore 560099, India

Full list of author information is available at the end of the article
}

oncogenesis [4-10]. Given the importance of PI3K signaling in cancer, several drugs targeting $\mathrm{p} 110 \alpha$ and/or other members of the p110 $\alpha$ family have been developed and are in clinical development [11]. Tumor suppressor TP53 is a commonly mutated cancer gene [2]. In human breast cancer, TP53 mutations occur in $37-46 \%$ of the cases $[1,2]$, and in about $13 \%$ of the cases, PIK3CA and TP53 mutations co-occur [1].

We previously developed a conditionally activatable Pik3caH1047R;MMTV-Cre mouse model where we found expression of Pik3caH1047R from the endogenous locus

(c) The Author(s). 2019 Open Access This article is distributed under the terms of the Creative Commons Attribution 4.0 International License (http://creativecommons.org/licenses/by/4.0/), which permits unrestricted use, distribution, and 
using MMTV-Cre primarily led to the development of benign mammary fibroadenomas [7]. In addition to benign tumors, we also found a few malignant sarcomatoid (spindle cell) carcinomas that had acquired spontaneous dominantnegative Trp53 mutations [7]. Given this and also the cooccurrence of PIK3CA and TP53 mutations in human breast cancers, we developed a Pik3caH1047R;Trp53R270H; MMTV-Cre double mutant model by crossing the Pik3caH1047R;MMTV-Cre mice with Trp53R270H flox mice [12]. We found that the Pik3caH1047R;Trp53R270H; MMTV-Cre double mutant mice had a shorter latency of 36.6 weeks for tumor development compared to 62 weeks in Pik3caH1047R;MMTV-Cre mice. Some of the double mutant animals also developed metastasis. Here, we report S100a4 to be a candidate gene involved in metastasis in this model.

\section{Methods}

Analysis of PIK3CA and TP53 mutations in human cancers PIK3CA and TP53 alterations were analyzed in 111,176 patient tumor samples, including 4485 breast cancer samples, sequenced with comprehensive genomic profiling (CGP) [13]. CGP was performed in a Clinical Laboratory Improvement Amendments (CLIA)-certified, CAP (College of American Pathologists)-accredited laboratory (Foundation Medicine, Inc., Cambridge, MA, USA) on all-comers during the course of routine clinical care. Approval was obtained from the Western Institutional Review Board (Protocol No. 20152817). Hybrid capture was performed for all coding exons from 285 to 315 cancer-related genes plus select introns from 28 genes frequently rearranged in cancer. We assessed all classes of genomic alterations (GA) including short variant, copy number, and rearrangement alterations, as previously described [9]. All known or likely pathogenic alterations were included in this analysis.

\section{Generation of Pik3caH1047R;Trp53R270H;MMTV-Cre mice}

Pik3caH1047R; MMTV-Cre mice were crossed with Trp53R270Hflox mice obtained from Dr. Tyler Jacks, Massachusetts Institute of Technology [12], to generate Pik3caH1047R;Trp53R270H;MMTV-Cre mice (Fig. 1a). Compound mutant mice were generated on $\mathrm{C} 57 \mathrm{BL} / 6$ genetic background. All mice were maintained in our animal facility as per the Institutional Animal Care and Use Committee (IACUC) guidelines. Mice were palpated weekly for the presence of tumors. Mice that showed any body condition score $<2$, a hunched posture, and $>$ $20 \%$ loss of body weight (together designated as underconditioned mice) were euthanized as per IACUC guidelines. Our study endpoint included mice with tumors that reached $\sim 2500 \mathrm{~mm}^{3}$ and the under-conditioned situation mentioned above.
Histological and immunofluorescence analysis

Five-micrometer, formalin-fixed, paraffin-embedded specimens were used for routine hematoxylin and eosin $(\mathrm{H} \& \mathrm{E})$ staining and histology evaluation. Immunofluorescence staining was performed using $10-\mu \mathrm{m}$ sections Tissue-Tek OCT (Sakura Finetek, CA) embedded frozen samples. Sectioned samples were fixed in $4 \%$ paraformaldehyde for $10 \mathrm{~min}$ and then blocked for $30 \mathrm{~min}$ with PBT (PBS with 0.1\% Triton) containing 1\% BSA. The blocked sections were then stained with appropriate primary antibody diluted in PBT with $0.1 \%$ BSA overnight at $4{ }^{\circ} \mathrm{C}$ in a humidified chamber. The slides were washed three times in PBT and then incubated with appropriate secondary antibodies for $60 \mathrm{~min}$ at room temperature in a humidified chamber. Unbound secondary antibody was removed by washing with PBT. Prolong Gold anti-fade reagent (Thermo Scientific, CA) was used to mount the slides. Primary antibodies used in the study were cytokeratin 5 (ab53121, Abcam, MA), cytokeratin18 (ab668, Abcam, MA), ER $\alpha$ (Ab-21, Thermo Scientific, CA), progesterone receptor (ab2764, Abcam, MA), and vimentin (ab45939, Abcam, MA). Appropriate secondary antibodies conjugated with Alexa 488 or 647 (Molecular probes, OR) were used for detecting the bound primary antibody.

\section{Development of primary tumor cell lines}

Tumor tissue was digested in PBS with $4 \mathrm{mg} / \mathrm{ml}$ collagenase (C0130-1G, Sigma, MO) and $5 \mathrm{mg} / \mathrm{ml}$ hyaluronidase (H2126-1G, Sigma, MO) for $1 \mathrm{~h}$ at $37^{\circ} \mathrm{C}$ to generate a cell suspension. Cells were then cultured in Epicult B basal medium (05610, Stemcell Technology, BC, Canada) with MEGM mammary epithelial cell growth medium containing SingleQuots supplements for growth factors (CC-4136, Lonza, MD), $1 \mathrm{ng} / \mathrm{ml} \mathrm{bFGF}$ (MilliporeSigma, MA), and heparin (1:100 dilution; 07980, Stemcell Technology, BC, Canada).

\section{Gene expression analysis}

Microarray data was collected as previously described [7]. Data normalization and differential gene expression analysis of the Agilent microarray data was performed using limma [14]. Metastatic sarcomatoid carcinoma group used in the expression analysis consisted of a Pik3caH1047R; Trp53R270H;MMTV-Cre primary tumor (tumor 1), a lung metastatic tumor (tumor 2) derived by tail vein injection involving a cell line derived from tumor 1, a Pik3caH1047R;MMTV-Cre primary tumor (tumor 3) with a spontaneous $\operatorname{Trp53R} 245 \mathrm{H}$ mutation previously described [7], a lung metastatic tumor (tumor 4) derived by tail vein injection involving a cell line derived from tumor 3 , and a Trp53R270H;MMTV-Cre primary tumors (tumor 5). Five adenocarcinomas Pik3caH1047R;Trp53R270H;MMTVCre primary tumors were also used in the expression 


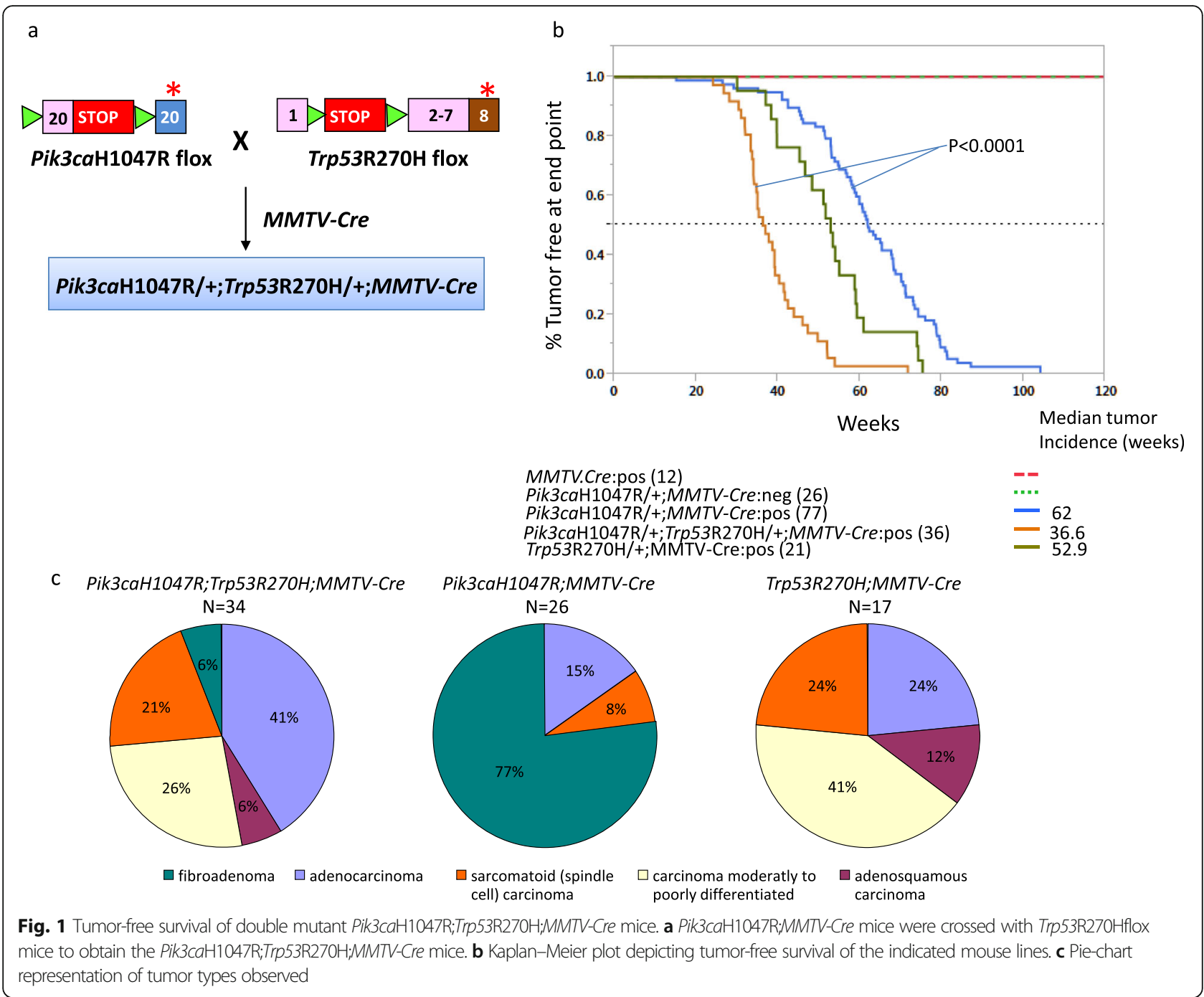

analysis. Cell lines developed from the adenocarcinoma were not metastatic in the tail vein injection model.

RNA-seq libraries were prepared using the TruSeq RNA Sample Preparation kit (Illumina, CA). The libraries were multiplexed three per lane and sequenced on the HiSeq platform to obtain, on average, $\sim 68$ million paired-end $(2 \times 75$-bp) reads per sample. All sequencing reads were evaluated for quality using the Bioconductor ShortRead package.

\section{Single-cell RNA-seq}

Single-cell suspension from the tumors was obtained using gentleMACS ${ }^{\mathrm{mm}}$ Dissociator (Milteny, Germany) and the associated tumor dissociation protocol. Single-cell RNA-seq data were generated using 10x Genomics Chromium Single Cell 3' kits (v1 \& v2). The cell density and viability of single-cell suspension was determined using a Vi-CELL (Beckman Coulter, CA) cell counter. The cDNA and libraries were prepared following the manufacturer's (10x genomics, $\mathrm{CA}$ ) instructions. Each library was quantified (Bioanalyzer High Sensitivity DNA kit (Agilent, CA) and Kapa Library quantification (Kapa Biosystems, MA) kit and sequenced on HiSeq2500/4000 (Illumina, CA).

\section{Single-cell RNA-seq data processing and analysis}

Single-cell RNA-seq data were processed with a custom pipeline. Briefly, reads were demultiplexed based on perfect matches to expected cell barcodes. Transcript reads were aligned to the mouse reference genome (GRCm38) using GSNAP (2013-10-10) only considering uniquely mapped reads [15]. Per-gene transcript counts were determined based on the number of unique UMIs for mapped reads overlapping exons in sense orientation, allowing for one mismatch when collapsing UMI sequences. To be considered for downstream analysis, cells were required to exceed a minimum number of detected transcripts, where a samplespecific cutoff was set to 0.1 times the total transcript count 
for cells at rank 30 (the 99th percentile for 3000 cells). Pergene transcript counts were normalized by dividing counts for each cell by a cell-specific scale factor, calculated as the total transcript count for a given cell, divided by the median total transcript count across cells. Normalized counts were then transformed using a $\log 2(\mathrm{x}+1)$ transformation. PCA was performed on the 1000 most variable genes. The top 20 principal components were used for model-based clustering with the R software package mclust (5.3) [16]. The final mixture model (VEV) and number of clusters $(n=19)$ were chosen based on minimal BIC score when considering up to 20 clusters. We identified genes enriched for each cluster using a non-parametric Wilcoxon rank-sum test. After inspecting enriched genes, clusters were merged into superclusters corresponding to epithelial cells $(n=2313)$, stromal cells $(n=369)$, T cells $(n=638)$, monocytes/macrophages $(n=1423)$, and putative cell multiplets $(n=252)$. Cell multiplets were not included in further analysis. Cells were also analyzed for expression of EMT genes (Snai1, Twist1, Zeb1, Zeb2) and S100a4.

\section{S100a4 CRISPR/Cas9 knockout cell lines}

A lentiviral sgRNA (guide RNA) and Cas9 expression vector, pLKO_SHC201_AIO_CMV_Cas9Puro, was constructed by gene synthesis (Genscript Inc., NJ) followed by cloning into the pLKO.5 backbone (Sigma-Aldrich, MO). sgRNAs targeting mouse S100a4 were identified as previously described [17]. The sgRNA targeting sequences are S100a4-2: 5'gctcaaggagctactgacc-3' (exon2) and S100a4-3: 5' -gacaatgaagttgacttcc- $3^{\prime}$ (exon3). sgRNAs were individually synthesized and cloned into pLKO_SHC201_AIO_CMV_Cas9Puro (Genscript Inc., NJ).

To generate lentivirus, low-passage HEK-293 cells were transiently transfected with a combination of $\mathrm{pLKO}_{-}$ SHC201_AIO_CMV_Cas9PURO_flox_gRNA expression plasmid, $\Delta 8.9$ packaging plasmid, and VSVG envelop plasmid using Lipofectamine 2000 (Life Technologies, CA) in Opti-MEM I media (Life Technologies, CA). After 72 h, lentiviruses were concentrated from cultured supernatants with Lenti-X Concentrator (Clontech, CA). Cell lines were infected with lentivirus and $8 \mu \mathrm{g} / \mathrm{ml}$ polybrene by two rounds of spin infection and selected on $2 \mu \mathrm{g} / \mathrm{ml}$ puromycin. Gene knockout was confirmed by aligning RNA-seq reads to the mouse genome (GRCm38) using GSNAP [15]. Allele frequencies of indels were computed by counting every indel event in reads aligning to the on-target position.

\section{Cell proliferation assay}

For each knockout line, $\sim 5000$ cells in 12 replicates were plated in a 384-well plate (Corning 3595, CA) coated with Collagen I. Cells were grown at $37^{\circ} \mathrm{C}$ in the presence of $5 \%$ $\mathrm{CO}_{2}$ and imaged at $\times 10$ magnification using an IncuCyte Zoom Live-content imaging system (Essen Bioscience, MI). Images were acquired every $2 \mathrm{~h}$ for 4 days, with four images per well. Data was analyzed using IncuCyte analysis software to detect and quantify live cell confluence (phase-contrast). Averages with standard error of the mean at each time point were plotted.

\section{Experimental metastasis assay}

Tumor-derived cell cultures were harvested at $70 \%$ confluency, counted, and dispersed in Hanks Balanced Buffer Solution (HBSS) at a concentration of 1 million cell per microliter. Target mouse was held with a mouse restrainer, with its tail sticking out of the small opening in the back of the restrainer. About 200,000 cells in a $200 \mu \mathrm{l}$ were injected into the mouse tail vein using a $1-\mathrm{ml}$ syringe. For the initial metastatic potential using tail vein injection, we analyzed three adenocarcinoma and three sarcomatoid carcinoma cell lines. We used three mice per line (a total of 18 mice) for this study. Mice were monitored for weight loss and labored breathing for 3 months. Lung tissue was harvested at the end of the study or when animals were euthanized under the study protocol guidelines. Gross lung examination was performed for the presence of metastasis, and metastasis was confirmed further by histological analysis. Metastasis studies were performed using the CRISPR knockout lines in a similar manner.

\section{Results}

\section{Co-occurrence of PIK3CA and TP53 mutations}

We observed spontaneous Trp53 mutations in a Pik3ca-mutant-driven mouse model of breast cancer that we had previously developed [7]. In an effort to understand this further, we assessed the co-occurrence of PIK3CA and TP53 in human cancers in a dataset of 111,176 patients involving 56 major cancer types [13]. Overall TP53 was mutated in 53\% of the samples and PIK3CA was mutated in $13 \%$ of the samples. In primary breast cancers, $62 \%(2763 / 4485)$ of the samples carried a TP53 mutation, making it the most frequently mutated gene. This was followed by PIK3CA which was mutated in $31 \%(1389 / 4485)$ of the breast cancer samples (Additional file 1: Figure S1). Overall, we found 15\% (678/4485) of the breast cancers carried both PIK3CA and TP53 mutations (Additional file 1: Figure S1B). Specifically, 49\% (678/1389) of the breast cancer samples with PIK3CA mutation also carried a TP53 mutation (Additional file 1: Figure S1C). This suggested that the two genes may function cooperatively in breast cancers.

\section{Pik3caH1047R;Trp53R270H;MMTV-Cre mice develop malignant mammary tumors with a shorter latency}

To understand the cooperativity between Pik3caH1047R and Trp53, we generated double mutant mice Pik3caH1047R;Trp53R270H;MMTV-Cre by crossing Pik3caH1047R;MMTV-Cre with Trp53 R270H flox mice (Fig. 1a). Trp53R270H mutation in mouse is equivalent to the human TP53R273H dominant-negative DNA- 
binding domain hotspot mutation. We validated the expression of the Pik3caH1047R and Trp53R270H in Pik3caH1047R;Trp53R270H;MMTV-Cre mammary tumors by RNA-seq (Additional file 1: Figure S2). The Pik3caH1047R;MMTV-Cre mice and the Trp53 R270H; $M M T V$-Cre mice had median tumor-free survival time of 62 weeks and 52.9 weeks, respectively. However, the double mutant mice Pik3caH1047R;Trp53R270H; $M M T V$-Cre showed a much shorter latency with the median tumor-free survival time of 36.6 weeks $(P<$ 0.0001; Fig. 1b). Consistent with this, in a limited set of animals, we observed the time to tumor survival from the initial detection was rapid in the Pik3caH1047R; Trp53R270H;MMTV-Cre double mutant mice compared to the single mutant mice (Additional file 1: Figure S3).

The Pik3caH1047R;MMTV-Cre animals generally develop benign fibroadenomas (77\%) [7]. In contrast, we found the
Pik3caH1047R;Trp53R270H;MMTV-Cre double mutant mice developed primarily mammary adenocarcinomas (41\%; Figs. 1c and 2). Additionally, they also developed moderate to poorly differentiated carcinomas (26\%) and sarcomatoid (spindle cell) carcinomas (21\%; Figs. 1c and 2). Histological analysis revealed that $15 \%(5 / 34)$ of the double mutant tumors had invasive features (Fig. 2a and Additional file 1: Figure S4A). Also, some tumors showed lymphocyte infiltration (Fig. 2e). The Trp53R270H;MMTV-Cre model also developed similar tumor types with the exception that no fibroadenomas were observed (Fig. 1c and Additional file 1: Figure S5). Among Pik3caH1047R;Trp53R270H;MMTV-Cre animals, we found an animal that developed sarcomatoid adenocarcinoma with lung metastasis (Fig. 2g and Additional file 1: Figure S4G). We examined Pik3caH1047R; MMTV-Cre mice $(n=4)$, Pik3caH1047R; Trp53R270H; $M M T V$-Cre mice $(n=9)$, and Trp53R270H;MMTV-Cre
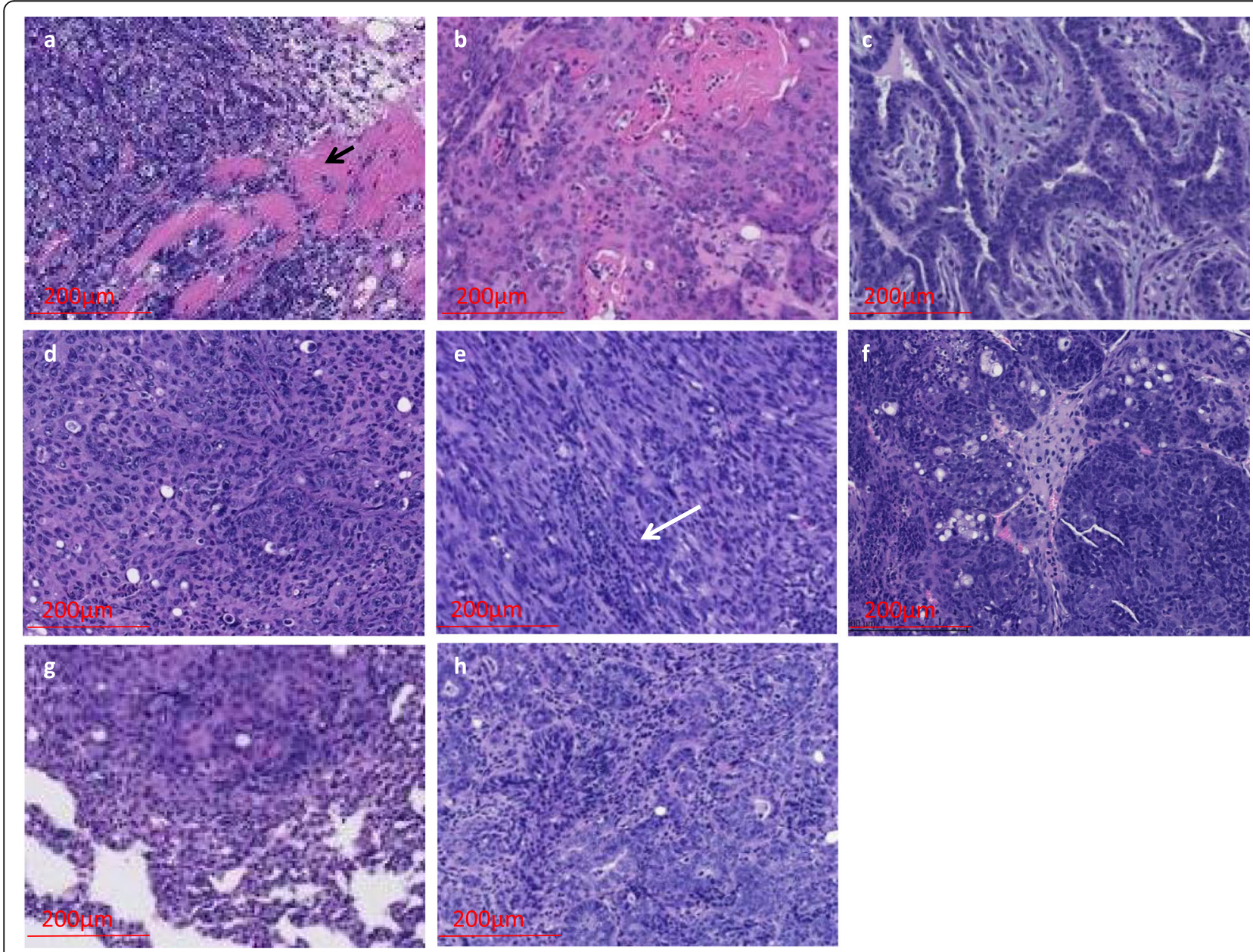

Fig. 2 Histology of Pik3caH1047R;Trp53R270H;MMTV-Cre primary mammary tumors and metastasis. a Invasive poorly differentiated adenocarcinoma (arrow indicates muscle fibers), $\mathbf{b}$ adenosquamous carcinoma, c fibroadenoma, $\mathbf{d}$ sarcomatoid adenocarcinoma, e sarcomatoid (spindle cell) carcinoma with lymphocyte infiltration (white arrow indicates site of lymphocyte infiltration), $\mathbf{f}$ poorly differentiated carcinoma, $\mathbf{g}$ metastatic lung carcinoma (from the same animal for which primary tumor is shown in panel $\mathbf{d}$ ), and $\mathbf{h}$ metastatic lymph node (from the same animal for which primary tumor is shown in panel e) 
mice $(n=2)$ for lymph node metastasis. We found evidence for lymph node metastasis in one Pik3caH1047R; Trp53R270H;MMTV-Cre animal with sarcomatoid (spindle cell) carcinoma (Fig. 2h and Additional file 1: Figure S4H). Overall, $\sim 21 \%$ (7/34) of the double mutant mice showed tumors with invasive or metastatic features. Also, one Trp53R270H animal with poorly differentiated carcinoma had metastasis in eight out of nine lymph nodes (Additional file 1: Figure S5F), suggesting that the Trp53 mutation likely contributes to the development of metastasis.

To understand the different cell types in tumors, we tested the expression of basal (Krt5), luminal (Krt18), and mesenchymal (Vimentin) markers using immunofluorescence. We observed both luminal and basal cells and also mesenchymal cells in the tumors (Additional file 1: Figure S6). We also tested the tumors for progesterone receptor (Pgr) and estrogen receptor (Esr) positivity. One Pik3caH1047R;Trp53R270H;MMTV-Cre poorly differentiated carcinoma showed a few Pgr-positive cells and was Esr negative, and another Pik3caH1047R;Trp53R270H; MMTV-Cre sarcomatoid adenocarcinoma showed only a few Esr-positive cells (Additional file 1: Figure S6C and 6E). A Trp53R270H;MMTV-Cre sarcomatoid carcinoma (Additional file 1: Figure S6F) was Esr negative (data not shown).

\section{Single-cell RNA-seq identifies distinct cell types in Pik3caH1047R;Trp53R270H;MMTV-Cre mammary tumors}

In order to further understand the cell types in tumors, we performed single-cell RNA-seq analysis on five tumor samples, including one Pik3caH1047R;MMTV-Cre tumor and four Pik3caH1047R;Trp53R270H;MMTV-Cre tumors. Principal component analysis (PCA) of single-cell RNA-seq data from 4995 total cells identified four distinct cell clusters in the tumors. They each predominantly expressed genes that are either epithelial, stromal, myeloid (monocytes and macrophages), or T cell types (Fig. 3a). The PCA profiles of the five individual tumors that included a benign fibroadenoma (Pik3caH1047R;Trp53R270H;MMTV-Cre; Figs. 3b and 2c), a metastatic sarcomatoid adenocarcinoma (Pik3caH1047R; Trp53R270H;MMTV-Cre; Figs. 3c and 2d), a malignant poorly differentiated carcinoma (Pik3caH1047R;Trp 53R270H;MMTV-Cre; Figs. 3d and 2f), and two other poorly differentiated carcinomas (Pik3caH1047R;Trp53R270H; MMTV-Cre; Fig. 3e and Pik3caH1047R;MMTV-Cre Fig. 3f) showed different proportions of cell types (Fig. 3b-f). Epithelial-mesenchymal transition (EMT) plays a central role in the multistep cascade leading to metastasis [18]. We assessed single-cell RNA-seq data for EMT-positive cells using an EMT score that is based on known EMT genes (Snai1, Twist1, Zeb1, and Zeb2; Additional file 1: Figure S7B). We found more cells undergoing EMT in the two malignant tumors compared to the fibroadenoma (37-38\% vs $11 \%$; Additional file 1: Figure S7).

\section{Identification of metastasis genes in}

\section{Pik3caH1047R;Trp53R270H;MMTV-Cre mammary tumors}

In order to understand the lung/lymph node metastatic phenotype observed in the double mutant Pik3caH1047R;Trp53R270H;MMTV-Cre tumors, we established cell lines from primary sarcomatoid (spindle type) carcinomas. We also established cell lines from adenocarcinomas. The cells derived from the sarcomatoid tumors showed spindle morphology (Fig. 4a), while the cells derived from adenocarcinomas were cobblestonelike (Fig. 4b). We tested the metastatic potential of these cell lines using tail vein injection in mice and found that while the adenocarcinoma-derived cell lines did not metastasize, several spindle-like cell lines metastasized to the lung (Fig. 4c, d). While mice injected with three different adenocarcenoma cell lines (3 mice per line) all had normal lungs at 3 months post-injection, $50 \%$ of the mice that received three different sarcomatoid carcinoma cell lines (3 mice per line) developed lung nodules within 55 days and all had developed nodules by 74 days. An average of 3 visible nodules per $1 \mathrm{~mm}^{2}$ was present as assessed by histological analysis.

To understand the metastatic sarcomatoid carcinoma and non-metastatic adenocarcinomas, we analyzed their gene expression (Fig. 4e). We found epithelial marker genes, Krt18, Epcam, Dsp, were downregulated in the metastatic tumors, while genes that promote EMT, Zeb2, Loxl2, and Plau were upregulated. Interestingly, $B M P 7$, an antagonist to EMT, which inhibits breast cancer metastasis [19], was a top gene that was downregulated in metastatic tumors. Also, pro-tumorigenic macrophage marker gene CD206 (Mrc1) and myeloid marker gene CD11b (Itgam) on average was $2.8 \times$ and $2.4 \times$ higher, respectively, in the metastatic tumors compared to non-metastatic carcinomas. Further, S100a4 and Arsj were among the top overexpressed genes in the metastatic tumors. Arsj is a sulfatase and its precise role in metastasis requires further investigation.

In this study, we further investigate the role of S100a4, a gene previously reported to be differentially expressed in highly metastatic mouse mammary carcinomas [20]. In humans, S100A4 is expressed during embryonic development in mesenchymal tissues, fetal macrophages, and trophoblasts, while in the adult stage, the expression is mainly restricted to immune cells such as macrophages, neutrophils, certain types of lymphocytes, and endothelial cells [21]. Analysis of S100A4 expression in human breast cancers showed that its levels were higher in tumors carrying PIK3CA H1047R or TP53 R270H or both compared to tumors without these mutations (Additional file 1: Figure S8). Previous studies have reported overexpression and association of S100A4 with tumor aggressiveness, metastasis, and poor survival in patients in multiple cancers [22, 23]. 


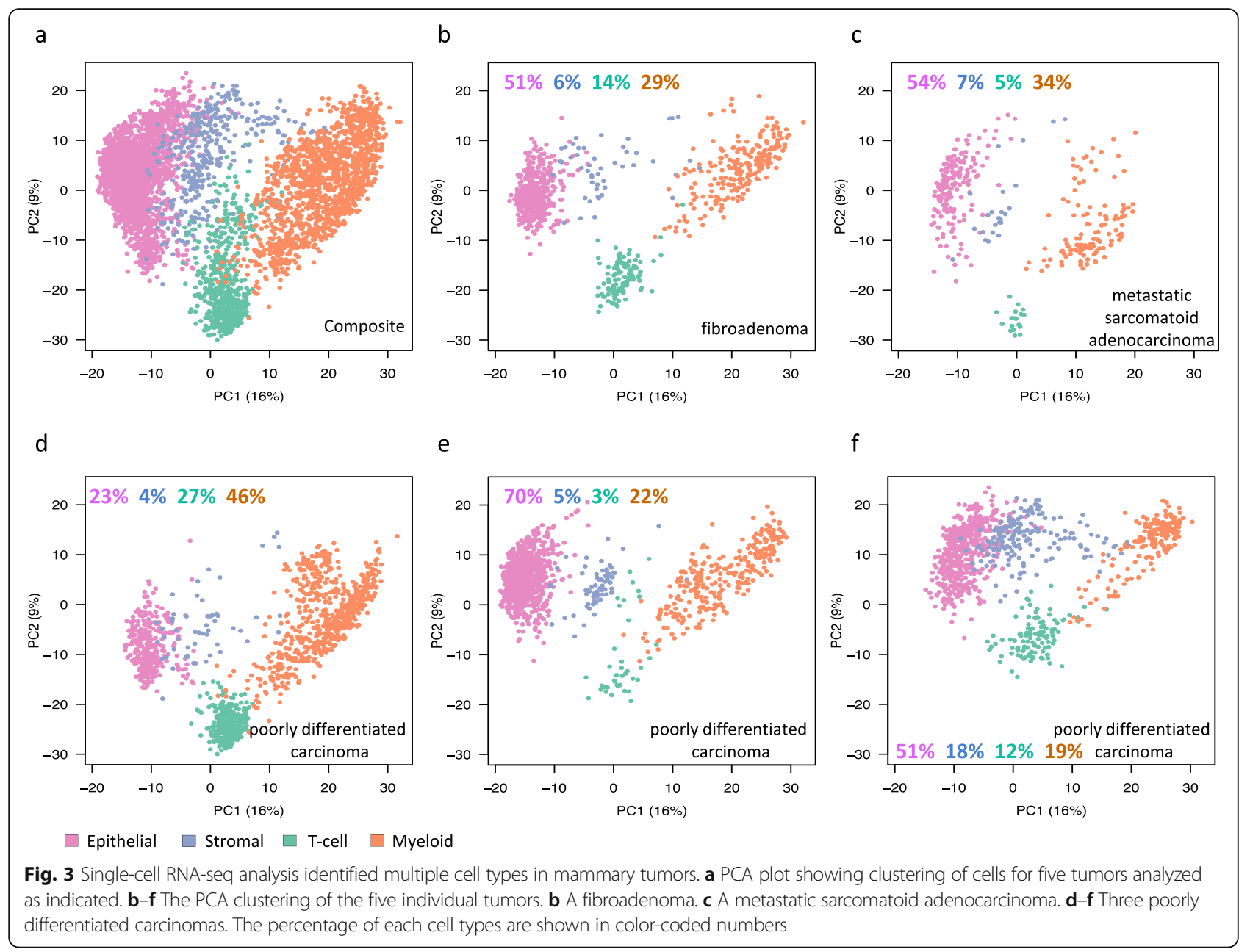

We assessed the expression of S100a4 in our singlecell RNA-seq data and found that all the three tumors and their constituent cell groups, epithelial, stromal, myeloid, and T cells, expressed S100a4 (Additional file 1: Figure S7C). Moreover, we found more S100a4 expressing cells in the two malignant tumors compared to fibroadenoma (51-56\% vs 26\%; Additional file 1: Figure S7C).

\section{CRISPR/Cas9 knockout S100a4 gene adversely affects metastatic potential}

The human S100A4 gene composed of four exons, with exons 3 and 4 encompassing the protein coding sequence, is located on chromosomal region 1q21. In mouse, S100a4 spans three exons where the first exon encodes the $5^{\prime}$ (untranslated region) UTR. S100a4 encodes a 101 amino acid protein that belongs to the S100 $\mathrm{Ca}^{2+}$ binding family (Fig. 5a). We knocked out S100a4 using two lentiviral constructs expressing S100a4 sgRNA1, corresponding to exon 2, and sgRNA2, corresponding to exon 3 (Fig. 5a). A cell line established from the primary tumor in the Pik3caH1047R;Trp53R270H; $M M T V$-Cre double mutant mice with lung metastasis was used to generate the knockout lines. Using RNAseq, we found evidence for the presence of protein truncating mutation in S100a4 (Fig. 5b) in 93.2 to $99.9 \%$, of the transcripts (Additional file 1: Table S1). Also, we confirmed the S100a4 loss by Western blot analysis (Additional file 1: Figure S9). We assessed the effect of the S100a4 knockout on cell proliferation by live cell imaging and found that it did not affect cellular proliferation when compared to the parental wildtype control line (Fig. 5c).

We analyzed the gene expression profile of the S100a4 knockout lines and found many gene expression changes compared to the parental line and vector control line using RNA-seq (Additional file 1: Figure S10). The epithelial marker gene Epcam was upregulated in the knockout lines compared to the parental line. In contrast, Sox4, the master regulator of EMT, and additional EMT genes such as Met, Zeb2, Etv1, and Idh1 were downregulated. Also, several matrix metalloproteinase genes, $M m p 3, M m p 9$, and $M m p 16$; extracellular matrix genes, Col3a1, Lamc2, Loxl2, and Bgn; and genes for adhesion molecules, Cdh13, Itgb1, and Itgb8, were 

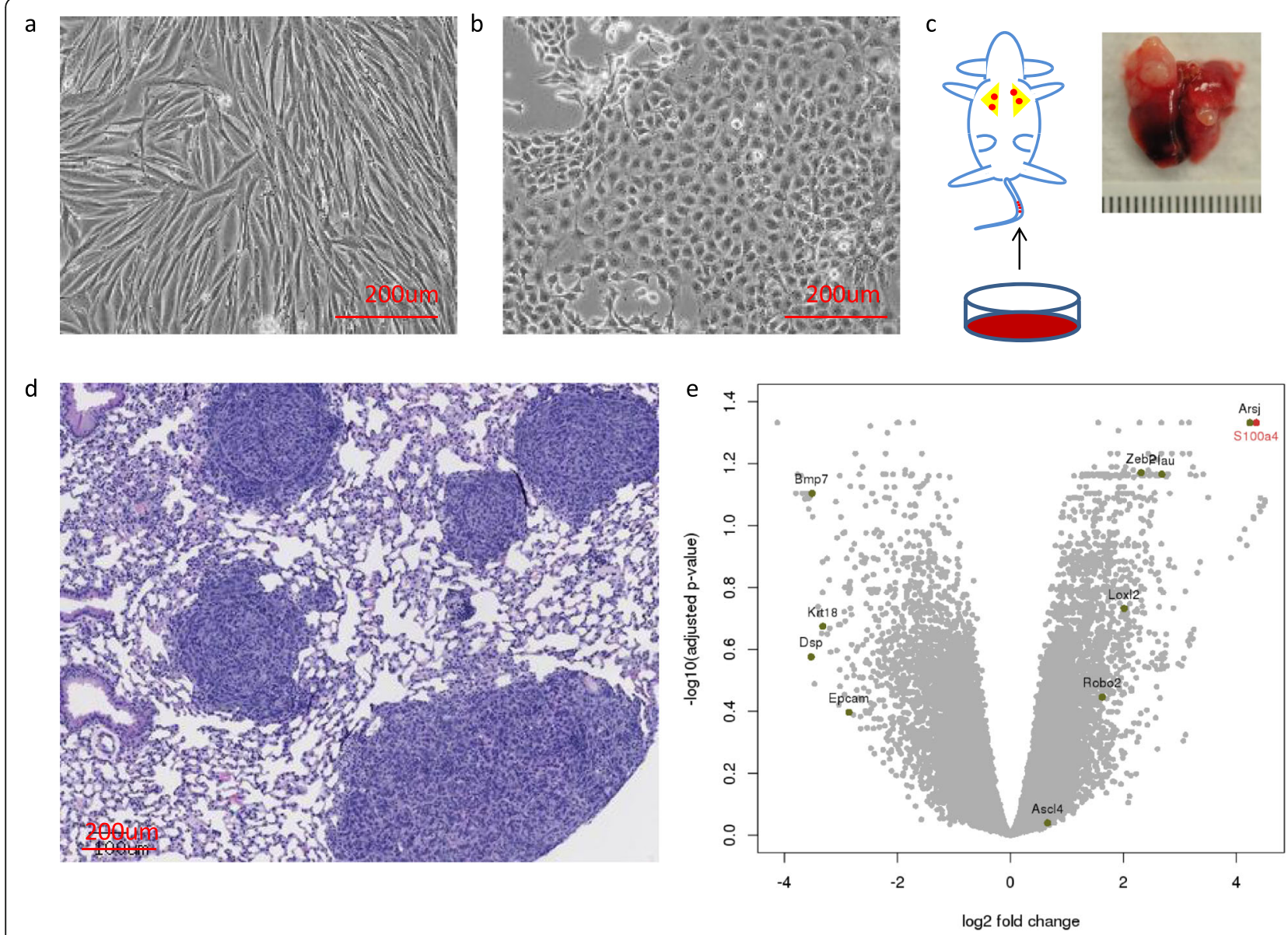

Fig. 4 Differential expression analysis identified metastasis-associated genes. a, b Tumor-derived cell lines with different $\mathbf{a}$ spindle- or $\mathbf{b}$ cobblestonelike epithelial morphology. $\mathbf{c}$ Schematic of the experimental metastasis assay. Lung with metastatic tumor nodules shown. $\mathbf{d}$ Histology of the lung with tumor nodules. e Volcano plot depicting the differentially expressed genes when comparing metastatic sarcomatoid carcinomas vs adenocarcinomas (see the "Methods" section)

downregulated. Interestingly, a long non-coding RNA gene Zfas1 was significantly upregulated in our S100a4 knockout lines. Previously, ZFAS1 was shown to be downregulated in human breast cancer cells and ZFAS1 overexpression led to reduced cell migration and invasion through inhibition of EMT [24]. Two chemokine genes, $\mathrm{Ccl} 2$ and $\mathrm{Ccl}$, which function in recruiting immune cells, were downregulated. In the knockout lines, several other pathways were affected, including P53 pathway (Mdm4, Crebbp, Kat2b), apoptosis pathway (Bcl2l1, Birc3, Cflar, Xiap, Traf1), Ras/Pi3k pathway (Braf, Jun, Pik3ca, Pik3r3, Pten, Sos2), Tgf $\beta$ pathway (Tgfb2, Tgfbr1, Smad5), Wnt pathway (Fzd9, Ctnnal1, Prkca, Wisp1), and hypoxia pathway (Hif1a). Myosin 1b, which regulates the actin crosslinking, is downregulated too. Interestingly, some genes that we previously found upregulated in the metastatic tumors, e.g., Arsj (Fig. 4), were also downregulated in the knockout lines (Additional file 1: Figure S10).
We tested the metastatic potential of the parental and knockout tumor cell lines using a syngeneic C57BL/6 tail vein injection model. We found that the parental tumor cell line developed from a Pik3caH1047R;Trp53R270H; $M M T V$-Cre metastatic tumor (Fig. 2d) when injected into mouse tail vein showed high metastatic potential (Fig. 4c, d). We found that compared to the parental line, mice receiving the S100a4 knockout lines did not develop lung nodules even until day 200 as assessed by necropsy (Fig. 5d). The mice receiving the parental or vector control line showed a marked reduction in survival $(n=4)$ and had developed lung nodules consistent with the expected metastatic potential of these lines.

\section{Discussion}

Genetically engineered mice (GEM) are powerful tools for understanding cancer development. In a set of 4485 breast cancer samples, we found PIK3CA (31\%) and TP53 (62\%) to be the top two mutated genes [13]. In this study, we assessed the consequence of breast cancer development in 


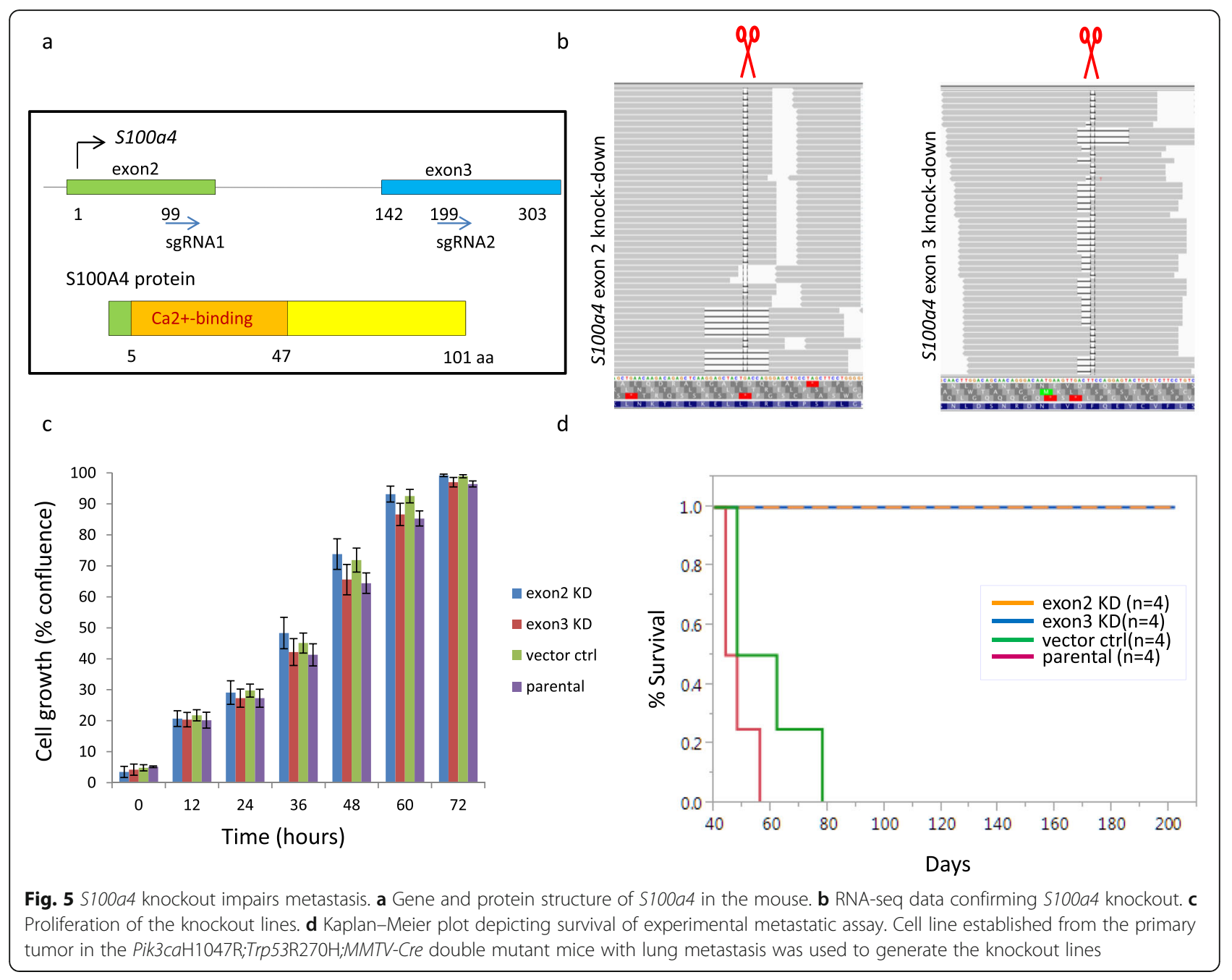

a mouse expressing Pik3caH1047R and a dominantnegative Trp53 mutant $\mathrm{R} 270 \mathrm{H}$. We found that the two genes cooperate in breast cancer development by drastically reducing the latency for tumor development (Fig. 1b). This is consistent with a previous study where transgenic Pik3ca mice, R26-Pik3caH1047R when crossed to a Trp53 null mouse, led to decreased survival [5].

Histological analysis identified multiple breast tumor types including adenocarcinoma, adenosquamous carcinoma, moderately to poorly differentiated carcinoma, and sarcomatoid (spindle cell) carcinoma. About $21 \%$ of the tumors were found to be sarcomatoid carcinoma. Similar diverse tumor histological tumor types were reported in a previous study [5]. In humans, metaplastic sarcomatoid carcinomas are mostly triple receptor-negative breast cancers (TNBC) and appear more aggressive than other TNBCs [25]. Metaplastic sarcomatoid carcinomas comprise a small but histologically diverse group of invasive breast cancers that share an unusual morphologic characteristic in that a part or all of the tumor cells appear to have undergone transformation to a non-glandular epithelial or mesenchymal cell type [26]. Also, there were increases in PIK3CA mutations, $A K T$ copy numbers, and phosphorylation of PI3K/AKT pathway components in metaplastic carcinomas compared to most other breast tumor subtypes [26]. Further, in our model, we found metastasis in a few animals with primary tumors displaying sarcomatoid features. Several cell lines derived from sarcomatoid tumors showed spindle cell morphology and were found to be metastatic. $M M T V$ PyMT, MMTV-Neu (Her2), MMTV-PyMT;Akt1-/-, $M M T V-P y M T ; C D 44-/-M M T V-C r e ; \quad T r p 53 \mathrm{fl} / \mathrm{fl}$, and $M M T V-C r e ; B r c a 1 f l / f l ; \operatorname{Trp} 53+/-$ mice were previously shown to develop lymph node and lung metastases [27, 28]. Additionally, WAP-Cre;Pten $\mathrm{fl} / \mathrm{fl} ; \quad \operatorname{Trp} 53+/$ $\mathrm{R} 270 \mathrm{H}$ mutant tumor cells were reported to show lung metastases [29]. Our model provides an additional system to study metastasis and develop therapeutic strategies for PIK3CA/TP53 double-positive cancers.

Single-cell RNA-seq analysis of the tumors identified epithelial, stromal, myeloid, and $\mathrm{T}$ cell types in the tumor. Metastatic tumors contained a much higher 
percentage of EMT-signature positive and S100a4-expressing cells among the epithelial, stromal, and myeloid clusters. Using CRISPR/CAS9-mediated knockout of S100a4, we demonstrate its relevance in metastasis. Genomic alterations at chromosomal region 1q21, in which most $S 100$ genes are clustered, are frequently observed in human epithelial tumors [21]. S100A4 is overexpressed in multiple cancers such as breast, lung, colorectal, esophageal, gastric, pancreatic, hepatocellular, gallbladder, and bladder cancers. Increased expression of S100A4 is associated with aggressiveness of a tumor, metastasis, and poor patient survival [22, 23]. Our analysis of S100A4 expression in human breast cancers showed that its levels were higher in tumors carrying PIK3CAH1047R or TP53R270H or both compared to tumors without these mutations (Additional file 1: Figure S8) though they were not statistically different. Given that the number of double mutant tumors with S100A4 expression data in this set (Additional file 1: Figure S8) is much lower than the single mutant tumors, analysis of a larger cohort of double mutant tumors with the tumor stage-specific information will help further confirm the role of S100A4 in metastasis. Overall, targeting S100A4 alone or in combination with driver mutations such as PIK3CA may provide a durable therapeutic intervention opportunity in aggressive breast cancers.

\section{Conclusions}

Pik3caH1047R;Trp53R270H;MMTV-Cre mouse described herein provides a preclinical model to mimic a subtype of human breast cancers and to investigate the genetic cooperation that drives primary mammary tumorigenesis and metastasis. S100a4, the critical molecule driving metastasis in this model, has the potential in diagnostics and therapeutic targeting.

\section{Supplementary information}

Supplementary information accompanies this paper at https://doi.org/10. 1186/s13058-019-1238-5.

Additional file 1: Figure S1. Frequency of TP53 and PIK3CA mutations in human breast cancer samples. A. TP53 and PIK3CA are the two most frequently altered genes in breast cancers. B. Frequency of PIK3CA and TP53 double mutant breast cancer samples. C. Frequency of TP53 mutations in breast cancer samples with PIK3CA mutations. Figure S2. Expression of Pik3caH1047R (A) and Trp53R270H (B) alleles in a Pik3caH1047R/+;Trp53R270H/+;MMTV-Cre tumor as assessed by RNA-seq Figure S3. Tumor progression in Pik3caH1047R;Trp53R270H;MMTV-Cre mice, Pik3caH1047R;MMTV-Cre mice and Trp53R270H;MMTV-Cre mice. Figure S4. Lower magnification of the panels in Fig. 2 in the same order. Figure S5. Histology of Trp53R270H;MMTV-Cre primary mammary tumors. A. adenocarcinoma, B. adenosquamous carcinoma, C. sarcomatoid (spindle cell) carcinoma, D. invasive poorly differentiated carcinoma on bone (arrow indicates bone tissue), E. poorly differentiated carcinoma, and F. metastatic lymph node (from the same animal bearing primary tumor in panel E). Figure S6. Immunofluorescence staining of a poorly differentiated Pik3caH1047R; Trp53R270H;MMTV-Cre carcinoma with focal sarcomatoid features (A-C) or sarcomatoid Pik3caH1047R;Trp53R270H;MMTV-Cre
(D-E) adenocarcinoma or sarcomatoid Trp53R270H:MMTV-Cre carcinoma (F). Staining for Krt18 (green), Krt5 (red), Vimentin (green), progesterone receptor (Pgr, green) and estrogen receptor(Esr, red) are shown. DAPI is shown in blue. Figure S7. Cellular heterogeneity of Pik3caH1047R;Trp53R270H;MMTV-Cre tumors. A. Same as Fig. 3B-D. EMT signature positive (B) and S100a4 positive (C) for cells in panel A. The numbers indicate the percentages of cell types (A), percentage of EMT positive cells (B) and S100a4 positive cells (C). Figure S8. Expression level of S100a4 in breast cancer TCGA data set (www.cbioportal.org). RSEM*: RNASeq by Expectation Maximization (BMC Bioinformatics 2011, 12:323). Figure S9. S100a4 protein levels in CRISPR/Cas9 knockout lines assessed by Western blotting. Figure S10. Log2 RPKM heatmap comparing RNA-seq based expression of select genes in the S100a4 knockout, parental and vector control cell lines. Table S1. Summary of allelic frequency, in the S100a4 knockout lines.

\section{Abbreviations}

MMTV: Mouse mammary tumor virus; EMT: Epithelial-mesenchymal transition; CGP: Comprehensive genomic profiling; CLIA: Clinical Laboratory Improvement Amendments; GA: Genomic alterations; IACUC: Institutional Animal Care and Use Committee; HBSS: Hanks Balanced Buffer Solution; UTR: Untranslated region; TNBC: Triple receptor-negative breast cancers

\section{Acknowledgements}

We thank Genentech animal facility and the DNA sequencing group for their help with the study.

Data

Expression data is available under GEO study accession GSE126351.

\section{Authors' contributions}

WY designed and performed experiments, analyzed the results, assembled all the data, and wrote the manuscript; LDG, SD, EWS, and TTN performed bioinformatics analysis; YC performed single-cell analysis; NMJ performed tail vein injection mouse studies; BH provided support on CRISPR studies; JZ provided pathology support; ZM supervised the sequence data collection; SS performed the study design and manuscript writing and editing; all the authors read, edited, and approved the manuscript.

\section{Funding}

Nothing to declare.

Availability of data and materials

Materials as appropriate will be made available.

Ethics approval and consent to participate

As stated in the methods and a previous publication (Nat. Biotechnol. 2013, 31:1023-1031), approval data was obtained from the Western Institutional Review Board (Protocol No. 20152817) for the human mutation data presented in the paper.

Consent for publication

All authors have approved the MS and consented for its publication.

\section{Competing interests}

The authors, as indicated, from Genentech, MedGenome or Foundation medicine hold shares/options in their respective organization.

\section{Author details}

'Department of Molecular Biology, Genentech Inc, 1 DNA Way, South San Francisco, CA 94080, USA. ²Department of Bioinformatics and Computational Biology, Genentech Inc, 1 DNA Way, South San Francisco, CA 94080, USA. ${ }^{3}$ Department of Cancer Signaling, Genentech Inc, 1 DNA Way, South San Francisco, CA 94080, USA. ${ }^{4}$ Foundation Medicine Inc., 150 Second Street, Cambridge, MA 02141, USA. ${ }^{5}$ Research and Development Department, MedGenome Inc., Foster City, CA 94404, USA. 'Department of Pathology, Genentech Inc, 1 DNA Way, South San Francisco, CA 94080, USA. ${ }^{7}$ SciGenom Research Foundation, Bangalore 560099, India. 
Received: 14 February 2019 Accepted: 6 December 2019

Published online: 27 December 2019

\section{References}

1. Yeang $\mathrm{CH}, \mathrm{McCormick} F$, Levine A. Combinatorial patterns of somatic gene mutations in cancer. FASEB J. 2008;22(8):2605-22.

2. TCGA. Comprehensive molecular portraits of human breast tumours. Nature. 2012;490(7418):61-70.

3. Samuels Y, Wang Z, Bardelli A, Silliman N, Ptak J, Szabo S, Yan H, Gazdar A, Powell SM, Riggins GJ, et al. High frequency of mutations of the PIK3CA gene in human cancers. Science. 2004;304(5670):554

4. Liu P, Cheng H, Santiago S, Raeder M, Zhang F, Isabella A, Yang J, Semaan DJ, Chen C, Fox EA, et al. Oncogenic PIK3CA-driven mammary tumors frequently recur via PI3K pathway-dependent and PI3K pathwayindependent mechanisms. Nat Med. 2011;17(9):1116-20.

5. Adams JR, Xu K, Liu JC, Agamez NM, Loch AJ, Wong RG, Wang W, Wright $\mathrm{KL}$, Lane TF, Zacksenhaus $\mathrm{E}$, et al. Cooperation between Pik3ca and p53 mutations in mouse mammary tumor formation. Cancer Res. 2011;71(7): 2706-17.

6. Meyer DS, Brinkhaus H, Muller U, Muller M, Cardiff RD, Bentires-Alj M. Luminal expression of PIK3CA mutant H1047R in the mammary gland induces heterogeneous tumors. Cancer Res. 2011;71(13):4344-51.

7. Yuan W, Stawiski E, Janakiraman V, Chan E, Durinck S, Edgar KA, Kljavin NM, Rivers CS, Gnad F, Roose-Girma M, et al. Conditional activation of Pik3ca(H1047R) in a knock-in mouse model promotes mammary tumorigenesis and emergence of mutations. Oncogene. 2013:32(3):318-26.

8. Tikoo A, Roh V, Montgomery KG, Ivetac I, Waring P, Pelzer R, Hare L, Shackleton M, Humbert P, Phillips WA. Physiological levels of Pik3ca(H1047R) mutation in the mouse mammary gland results in ductal hyperplasia and formation of ERalpha-positive tumors. PLoS One. 2012;7(5): e36924

9. Koren S, Reavie L, Couto JP, De Silva D, Stadler MB, Roloff T, Britschgi A, Eichlisberger T, Kohler $\mathrm{H}$, Aina O, et al. PIK3CA(H1047R) induces multipotency and multi-lineage mammary tumours. Nature. 2015;525(7567):114-8.

10. Van Keymeulen A, Lee MY, Ousset M, Brohee S, Rorive S, Giraddi RR, Wuidart A, Bouvencourt G, Dubois C, Salmon I, et al. Reactivation of multipotency by oncogenic PIK3CA induces breast tumour heterogeneity. Nature. 2015; 525(7567):119-23.

11. Yang Q, Modi P, Newcomb T, Queva C, Gandhi V. Idelalisib: first-in-class PI3K delta inhibitor for the treatment of chronic lymphocytic leukemia, small lymphocytic leukemia, and follicular lymphoma. Clin Cancer Res. 2015;21(7): 1537-42.

12. Olive KP, Tuveson DA, Ruhe ZC, Yin B, Willis NA, Bronson RT, Crowley D, Jacks T. Mutant p53 gain of function in two mouse models of Li-Fraumeni syndrome. Cell. 2004;119(6):847-60

13. Hartmaier RJ, Albacker LA, Chmielecki J, Bailey M, He J, Goldberg ME, Ramkissoon S, Suh J, Elvin JA, Chiacchia S, et al. High-throughput genomic profiling of adult solid tumors reveals novel insights into cancer pathogenesis. Cancer Res. 2017;77(9):2464-75.

14. Ritchie ME, Phipson B, Wu D, Hu Y, Law CW, Shi W, Smyth GK. limma powers differential expression analyses for RNA-sequencing and microarray studies. Nucleic Acids Res. 2015;43(7):e47.

15. Wu TD, Nacu S. Fast and SNP-tolerant detection of complex variants and splicing in short reads. Bioinformatics (Oxford, England). 2010;26(7):873-81.

16. Scrucca L, Fop M, Murphy TB, Raftery AE. mclust 5: clustering, classification and density estimation using Gaussian finite mixture models. R J. 2016;8(1):289-317.

17. Callow MG, Watanabe C, Wickliffe KE, Bainer R, Kummerfield S, Weng J, Cuellar T, Janakiraman V, Chen H, Chih B, et al. CRISPR whole-genome screening identifies new necroptosis regulators and RIPK1 alternative splicing. Cell Death Dis. 2018;9(3):261.

18. Bill $R$, Christofori $G$. The relevance of EMT in breast cancer metastasis: correlation or causality? FEBS Lett. 2015;589(14):1577-87.

19. Buijs JT, van der Horst $G$, van den Hoogen C, Cheung H, de Rooij B, Kroon J, Petersen M, van Overveld PG, Pelger RC, van der Pluijm G. The BMP2/7 heterodimer inhibits the human breast cancer stem cell subpopulation and bone metastases formation. Oncogene. 2012;31(17):2164-74.

20. Ebralidze A, Tulchinsky E, Grigorian M, Afanasyeva A, Senin V, Revazova E, Lukanidin E. Isolation and characterization of a gene specifically expressed in different metastatic cells and whose deduced gene product has a high degree of homology to a Ca2+-binding protein family. Genes Dev. 1989; 3(7):1086-93.
21. Lukanidin E, Sleeman JP. Building the niche: the role of the $\mathrm{S} 100$ proteins in metastatic growth. Semin Cancer Biol. 2012;22(3):216-25.

22. Bresnick AR, Weber DJ, Zimmer DB. S100 proteins in cancer. Nat Rev Cancer. 2015;15(2):96-109.

23. Fei F, Qu J, Zhang M, Li Y, Zhang S. S100A4 in cancer progression and metastasis: a systematic review. Oncotarget. 2017:8(42):73219-39.

24. Fan S, Fan C, Liu N, Huang K, Fang X, Wang K. Downregulation of the long non-coding RNA ZFAS1 is associated with cell proliferation, migration and invasion in breast cancer. Mol Med Rep. 2018;17(5):6405-12.

25. Lester TR, Hunt KK, Nayeemuddin KM, Bassett RL Jr, Gonzalez-Angulo AM, Feig BW, Huo L, Rourke LL, Davis WG, Valero V, et al. Metaplastic sarcomatoid carcinoma of the breast appears more aggressive than other triple receptornegative breast cancers. Breast Cancer Res Treat. 2012;131(1):41-8.

26. Hennessy BT, Gonzalez-Angulo AM, Stemke-Hale K, Gilcrease MZ, Krishnamurthy S, Lee JS, Fridlyand J, Sahin A, Agarwal R, Joy C, et al. Characterization of a naturally occurring breast cancer subset enriched in epithelial-to-mesenchymal transition and stem cell characteristics. Cancer Res. 2009;69(10):4116-24.

27. Kim IS, Baek SH. Mouse models for breast cancer metastasis. Biochem Biophys Res Commun. 2010;394(3):443-7.

28. Saxena $M$, Christofori $G$. Rebuilding cancer metastasis in the mouse. Mol Oncol. 2013:7(2):283-96

29. Wang S, Liu JC, Kim D, Datti A, Zacksenhaus E. Targeted Pten deletion plus p53-R270H mutation in mouse mammary epithelium induces aggressive claudin-low and basal-like breast cancer. Breast Cancer Res. 2016;18(1):9.

\section{Publisher's Note}

Springer Nature remains neutral with regard to jurisdictional claims in published maps and institutional affiliations.
Ready to submit your research? Choose BMC and benefit from:

- fast, convenient online submission

- thorough peer review by experienced researchers in your field

- rapid publication on acceptance

- support for research data, including large and complex data types

- gold Open Access which fosters wider collaboration and increased citations

- maximum visibility for your research: over $100 \mathrm{M}$ website views per year

At $\mathrm{BMC}$, research is always in progress.

Learn more biomedcentral.com/submissions 